\title{
Electron density of states and spectrum of disordered $s-d$ model
}

\author{
Yu.K.Rudavskii, G.V.Ponedilok, L.A.Dorosh \\ Lviv National Polytechnic University, \\ 12 S.Bandera Str., 79013 Lviv, Ukraine
}

Received August 23, 2000, in final form November 1, 2000

Structurally disordered s-d model of magnetism in metals is investigated. The simplified model of binary alloy structure is offered, the structural correlation functions are calculated. The self-consistent equations for calculation of a spectrum, magnetization, electronic spin polarization and temperature of phase transition are obtained.

Key words: amorphous systems, magnetization, polarization

PACS: $75.30 . D s, 75.50 . K$

\section{Introduction}

The exchange $s$ - $d$ model is used to describe the alloy properties, that contains transitional and rare-earth elements. The exchange $s$ - $d$ model offered by Shubin and Vonsovskii was further developed by Ziner, Turov, Kassuj, Iosid and others, and it found a further application in different fields of solid state physics. First the electric and magnetic properties description of transitional $d$-metals was suggested and at present it is a basic magnetism theory of the rare-earth metals, magnetic quasiconductors [1] and dissolved alloys of transitional metals [3]. From the theoretical point of view, the importance of the $s$ - $d$ model is determined by a lot of wonderful and non-trivial effects that it contains: formation of spin polarons, ferrons, fluctuons in magnetic quasi-conductors $[1,4]$, occurrence of the spin glass state in metallic alloys [2], Kondo effect etc.

\section{Spin-electron model of amorphous magnet}

An amorphous system of $N$ atoms in volume $V \subset \mathbb{R}^{3}$ is considered, part of which has got localized magnetic moments (further - magnetic alloy subsystem), and other atoms do not have localized magnetic moments (non-magnetic subsystem of amorphous alloy). Atomic coordinates $\left(\mathbf{R}_{1}, \ldots, \mathbf{R}_{N}\right)=\mathbb{R}^{N} \in V$ accept random values. Quantitative relation between magnetic and non-magnetic atoms is described by 
concentration $c(0 \leqslant c \leqslant 1)$. The microscopic model of amorphous magnet consists of two quantum subsystems i.e., a subsystem of the localized atomic spins and a subsystem of conductivity electrons.

Hamiltonian of model has the form

$$
\hat{H}=\hat{H}_{\mathrm{s}}+\hat{H}_{\mathrm{el}}+\hat{H}_{\mathrm{el}-\mathrm{s}} .
$$

The first term describes the energy of the subsystem of localized spins, which are in the external magnetic field $h$, and the pair couple by the Heisenberg exchange interaction

$$
\hat{H}_{\mathrm{s}}=\frac{1}{2} N c S(S+1) J(|\mathbf{R}|=0)-\mu h \sum_{1 \leqslant j \leqslant N} \hat{c}_{j} S_{j}^{z}-\frac{1}{2} \sum_{\mathbf{q} \in \Lambda} J_{\mathbf{q}} \mathbf{S}_{\mathbf{q}} \mathbf{S}_{-\mathbf{q}} .
$$

Here $\mu$ is the moment of magnetic atoms, and $\mathbf{S}_{j}$ is the spin operator of the magnetic atom placed in point $\mathbf{R}_{j} \in V$.

The subsystem of conductivity electrons is described in the pseudopotential approach

$$
\hat{H}_{\mathrm{el}}=\sum_{\mathbf{k} \in \Lambda} \sum_{\sigma= \pm 1} \mathcal{E}_{\mathbf{k} \sigma} a_{\mathbf{k}, \sigma}^{+} a_{\mathbf{k}, \sigma}+\sum_{\mathbf{k}, \mathbf{q} \in \Lambda} \sum_{\sigma \pm 1} W_{\mathbf{q}} a_{\mathbf{k}, \sigma}^{+} a_{\mathbf{k}-\mathbf{q}, \sigma}
$$

Here $a_{\mathbf{k} \sigma}^{+}\left(a_{\mathbf{k} \sigma}\right)$ are Fermi operators of creation (annihilation) of electrons in states $\{\mathbf{k}, \sigma\}$. The index $\sigma$ takes values respective to two possible projections of the electron spin on the axis of quantization $O Z$. In the paper we also employed notation $\sigma=(\uparrow$ $, \downarrow)$. The wave vector $\mathbf{k} \in \Lambda$, where $\Lambda=\left\{\mathbf{k}: \mathbf{k}=\sum_{1 \leqslant \alpha \leqslant 3} 2 \pi V^{-1 / 3} n_{\alpha} \mathbf{e}_{\alpha}, \quad n_{\alpha} \in \mathbb{Z}\right.$, $\left.\left(\mathbf{e}_{\alpha}, \mathbf{e}_{\beta}\right)=\delta_{\alpha \beta}\right\}$ is momentum quasi-continuous space. The spectrum of free electronic gas in presence of external magnetic field $h$ is

$$
\mathcal{E}_{\mathbf{k} \sigma}=\frac{\hbar^{2} k^{2}}{2 m}-\varkappa_{\sigma} \mu_{\mathrm{B}} h
$$

Scattering potential of the conductivity electrons on the ions is given by

$$
W_{\mathbf{q}}=\frac{1}{N} \sum_{j=1}^{N} \mathrm{e}^{-\mathrm{iq} \mathbf{R}_{j}} w_{\mathbf{q}}, \quad w_{\mathbf{q}}=\frac{N}{V} \int_{V} \mathrm{~d} \mathbf{r} w(|\mathbf{r}|) \mathrm{e}^{-\mathrm{iq} \mathbf{r}},
$$

where $w(|\mathbf{r}|)$ is an electron-ions pseudopotential. For local pseudopotentials, the matrix element of scattering potential of electrons on ions $w_{\mathbf{q}}$ depends only on the module of impulse transfer.

The Hamiltonian of spin-electron interaction takes the form

$$
\hat{H}_{\mathrm{el-s}}=-\frac{1}{\sqrt{N}} \sum_{\mathbf{q} \in \Lambda} I_{\mathbf{q}}\left\{S_{\mathbf{q}}^{z} \hat{\sigma}_{-\mathbf{q}}^{z}+\frac{1}{2}\left[S_{\mathbf{q}}^{+} \hat{\sigma}_{-\mathbf{q}}^{-}+S_{\mathbf{q}}^{-} \hat{\sigma}_{-\mathbf{q}}^{+}\right]\right\} \text {. }
$$

In expression (6), which is similar to (2), the operator

$$
S_{\mathbf{q}}^{\alpha}=\frac{1}{\sqrt{N}} \sum_{1 \leqslant j \leqslant N} \hat{c}_{j} S_{j}^{\alpha} \mathrm{e}^{-\mathrm{iq} \mathbf{R}_{j}}, \quad \alpha=z,+,-
$$


where $S_{j}^{ \pm}=S_{j}^{x} \pm \mathrm{i} S_{j}^{y}$. In the case of lattice systems $\left(\mathbf{R}_{j} \in \mathbb{Z}^{3}\right)$ the operator $(7)$ is the exact Fourier-transform of operators $S_{j}^{\alpha}$.

In formula (6) the bilinear combinations of electrons creation and annihilation operators are defined

$$
\begin{aligned}
& \hat{\sigma}_{\mathbf{q}}^{z}=\sum_{\sigma=\uparrow, \downarrow} \frac{\varkappa_{\sigma}}{2} \hat{n}_{\mathbf{q}, \sigma}, \quad \hat{n}_{\mathbf{q} \sigma}=\sum_{\mathbf{k} \in \Lambda} a_{\mathbf{k}, \sigma}^{+} a_{\mathbf{k}+\mathbf{q}, \sigma}, \\
& \hat{\sigma}_{\mathbf{q}}^{+}=\sum_{\mathbf{k} \in \Lambda} a_{\mathbf{k}, \uparrow}^{+} a_{\mathbf{k}+\mathbf{q}, \downarrow}, \quad \hat{\sigma}_{\mathbf{q}}^{-}=\sum_{\mathbf{k} \in \Lambda} a_{\mathbf{k}, \downarrow}^{+} a_{\mathbf{k}+\mathbf{q}, \uparrow} .
\end{aligned}
$$

The Fourier-components $\hat{n}_{\mathbf{q}}$ of the density operator are

$$
\hat{n}_{\mathbf{q}}=\int \mathrm{d} \mathbf{r} \hat{n}(\mathbf{r}) \mathrm{e}^{-\mathrm{iqr}} ; \quad \hat{n}(\mathbf{r})=\frac{1}{V} \sum_{\mathbf{q} \in \Lambda} \hat{n}_{\mathbf{q}} \mathrm{e}^{\mathbf{i q r}} .
$$

Hermitian operator $\hat{\sigma}_{\mathbf{q}}^{z}$ is the Fourier-component of the operator of the electronic spin polarization density.

It is convenient to describe the structure of amorphous system by correlation functions

$$
Q_{n}\left(\mathbf{k}_{1}, \ldots, \mathbf{k}_{n} ; c\right)=\left\langle\hat{c}_{\mathbf{k}_{1}} \ldots \hat{c}_{\mathbf{k}_{n}}\right\rangle_{\text {conf }}^{\text {irr }} .
$$

Here the Fourier-coefficient of the atomic density fluctuations is

$$
\hat{c}_{\mathbf{k}}=\frac{1}{\sqrt{N}} \sum_{j=1}^{N} \hat{c}_{j} \mathrm{e}^{-\mathrm{i} \mathbf{k} \mathbf{R}_{j}}, \quad \mathbf{k} \neq \mathbf{0} .
$$

The structure correlation functions are reduced to such a form

$$
Q_{1}(\mathbf{k} ; c)=0, \quad Q_{2}\left(\mathbf{k}_{1}, \mathbf{k}_{2} ; c\right)=c\left(1-c+c S_{2}\left(\mathbf{k}_{1},-\mathbf{k}_{1}\right)\right) \delta_{\mathbf{k}_{1}+\mathbf{k}_{2}, \mathbf{0}}, \quad \ldots .
$$

The correlation functions of the atomic density fluctuations $S_{m}\left(\mathbf{k}_{1}, \ldots, \mathbf{k}_{m}\right)$ are impossible to calculate from the first principles, and were accepted as phenomenological values.

Let us suppose that fluctuations of atomic density $\varrho_{\mathbf{k}}$ are described by the Gaussian law. Probability distribution function of fluctuations of the atomic density is

$$
P\left(\ldots, \varrho_{\mathbf{k}}, \ldots\right)=\prod_{\mathbf{k} \neq \mathbf{0}} \frac{1}{\sqrt{2 \pi S_{2}(k)}} \exp \left[-\sum_{\mathbf{k} \neq \mathbf{0}} \frac{\varrho_{\mathbf{k}} \varrho_{-\mathbf{k}}}{S_{2}(k)}\right] .
$$

Dispersion of Gaussian distribution

$$
S_{2}(k)=\int\left(\mathrm{d} \varrho_{\mathbf{k}}\right) \varrho_{\mathbf{k}} \varrho_{-\mathbf{k}} P\left(\ldots, \varrho_{\mathbf{k}}, \ldots\right)
$$

is the pair structural system factor. Integration in formula (13) over $\varrho_{\mathbf{k}}$ with distribution function (12) is equivalent to the operation of configurational averaging. The structural functions of higher order as follows:

$$
S_{m}\left(\mathbf{k}_{1}, \ldots, \mathbf{k}_{m}\right) \stackrel{\text { def }}{=}\left\langle\varrho_{\mathbf{k}_{1}}, \ldots \varrho_{\mathbf{k}_{m}}\right\rangle_{\mathbf{R}}=\int\left(\mathrm{d} \varrho_{\mathbf{k}}\right) \varrho_{\mathbf{k}_{1}} \cdots \varrho_{\mathbf{k}_{m}} P\left(\ldots, \varrho_{\mathbf{k}}, \ldots\right) .
$$


When $m$ is even $(m=2 n)$, then

$$
S_{2 n}\left(\mathbf{k}_{1}, \ldots, \mathbf{k}_{2 n}\right)=\sum_{\{p\}} S_{2}\left(\mathbf{k}_{i_{1}}\right) \delta_{\mathbf{k}_{i_{1}}+\mathbf{k}_{j_{1}}, \mathbf{0}} S_{2}\left(\mathbf{k}_{i_{2}}\right) \delta_{\mathbf{k}_{i_{2}}+\mathbf{k}_{j_{2}}, \mathbf{0}} \cdots S_{2}\left(\mathbf{k}_{i_{n}}\right) \delta_{\mathbf{k}_{i_{n}}+\mathbf{k}_{j_{n}}, \mathbf{0}}
$$

In this formula the sum is taken over for all possible decomposition wave numbers $\mathbf{k}_{1}, \ldots, \mathbf{k}_{2 n}$ on pairs.

\section{Dispersion law and density of electron states}

The Green function $G^{\sigma}(\mathbf{k}, \mathbf{q} \mid \omega)=\left\langle\left\langle a_{\mathbf{k}, \sigma} \mid a_{\mathbf{q}, \sigma^{\prime}}^{+}\right\rangle\right\rangle_{\omega}$ is needed to evaluate the distribution of electrons in momentum space

$$
n_{\mathbf{k}, \sigma}=\mathrm{i} \lim _{\varepsilon \rightarrow 0} \int_{-\infty}^{+\infty} \frac{G^{\sigma}(\mathbf{k}, \mathbf{k} \mid \omega+\mathrm{i} \varepsilon)-G^{\sigma}(\mathbf{k}, \mathbf{k} \mid \omega-\mathrm{i} \varepsilon)}{\mathrm{e}^{\beta \omega}+1} \mathrm{~d} \omega .
$$

Where $\beta=\left(k_{\mathrm{B}} T\right)^{-1}$ is the inverse temperature in energy units.

For Hamilton operator (1) the equation of motion for one-electron Green function has the form

$$
\begin{aligned}
& \left(\hbar \omega-\mathcal{E}_{\mathbf{k}, \sigma}\right) G^{\sigma}(\mathbf{k}, \mathbf{q} \mid \omega)=\frac{1}{2 \pi} \delta_{\mathbf{k}, \mathbf{q}}+\sum_{\mathbf{p} \in \Lambda} W_{\mathbf{p}} G^{\sigma}(\mathbf{k}-\mathbf{p}, \mathbf{q} \mid \omega) \\
& -\frac{1}{2 \sqrt{N}} \sum_{\mathbf{p} \in \Lambda} I_{\mathbf{p}}\left\{\varkappa_{\sigma} L^{\sigma}(\mathbf{p}, \mathbf{k}-\mathbf{p} ; \mathbf{q} \mid \omega)+M^{\sigma}(\mathbf{p}, \mathbf{k}-\mathbf{p} ; \mathbf{q} \mid \omega)\right\}, \sigma= \pm 1(\uparrow, \downarrow) .
\end{aligned}
$$

The equation (17) contains the Green functions of higher order

$$
L^{\sigma}(\mathbf{p}, \mathbf{l} ; \mathbf{q} \mid \omega)=\left\langle\left\langle S_{\mathbf{p}}^{z} a_{\mathbf{l}, \sigma} \mid a_{\mathbf{q}, \sigma}^{+}\right\rangle\right\rangle_{\omega}, \quad M^{\sigma}(\mathbf{p}, \mathbf{l} ; \mathbf{q} \mid \omega)=\left\langle\left\langle S_{\mathbf{p}}^{-\sigma} a_{\mathbf{l},-\sigma} \mid a_{\mathbf{q}, \sigma}^{+}\right\rangle\right\rangle_{\omega} .
$$

We introduce here the decoupling procedure

$$
L^{\sigma}(\mathbf{p}, \mathbf{k}-\mathbf{p} ; \mathbf{q} \mid \omega) \rightarrow\left\langle S_{\mathbf{p}}^{z}\right\rangle\left\langle\left\langle a_{\mathbf{k}-\mathbf{p}, \sigma} \mid a_{\mathbf{q}, \sigma}^{+}\right\rangle\right\rangle_{\omega}, \quad M^{\sigma}(\mathbf{p}, \mathbf{k}-\mathbf{p} ; \mathbf{q} \mid \omega) \equiv 0
$$

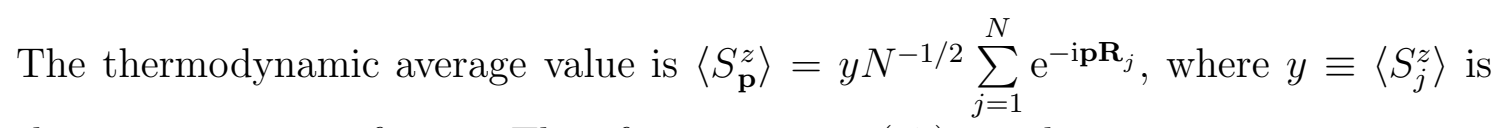
the magnetization of atom. Then from equation (17) we obtain

$$
\left(\hbar \omega-\mathcal{E}_{\mathbf{k}, \sigma}\right) G^{\sigma}(\mathbf{k}, \mathbf{q} \mid \omega)=\frac{1}{2 \pi} \delta_{\mathbf{k}, \mathbf{q}}+\sum_{\mathbf{p} \in \Lambda, \mathbf{p} \neq \mathbf{0}} \widetilde{W}_{\mathbf{p}, \sigma} G^{\sigma}(\mathbf{k}-\mathbf{p}, \mathbf{q} \mid \omega) .
$$

Here the effective scattering pseudopotential

$$
\widetilde{W}_{\mathbf{p}, \sigma} \equiv \frac{1}{N} \sum_{j=1}^{n}\left(w_{\mathbf{p}}-\frac{1}{2} I_{\mathbf{p}} y \varkappa_{\sigma}\right) \mathrm{e}^{-\mathrm{i} \mathbf{p} \mathbf{R}_{j}}=\frac{1}{N} \sum_{j=1}^{n} \widetilde{w}_{\mathbf{p}, \sigma} \mathrm{e}^{-\mathbf{i} \mathbf{R} \mathbf{R}_{j}}
$$


We write the following equation to calculate the configurational averaging of one-electron Green function

$$
\begin{aligned}
(\hbar \omega & \left.-\mathcal{E}_{\mathbf{k}-\mathbf{p}, \sigma}\right)\left\langle\widetilde{W}_{\mathbf{p}, \sigma} G^{\sigma}(\mathbf{k}-\mathbf{p}, \mathbf{q} \mid \omega)\right\rangle_{\mathrm{conf}}= \\
& =\frac{1}{2 \pi} \delta_{\mathbf{k}-\mathbf{p}, \mathbf{q}}\left\langle\widetilde{W}_{\mathbf{p}, \sigma}\right\rangle_{\mathrm{conf}}+\sum_{\mathbf{p}^{\prime} \in \Lambda}\left\langle\widetilde{W}_{\mathbf{p}, \sigma} \widetilde{W}_{\mathbf{p}^{\prime}, \sigma} G^{\sigma}\left(\mathbf{k}-\mathbf{p}-\mathbf{p}^{\prime}, \mathbf{q} \mid \omega\right)\right\rangle_{\mathrm{conf}} .
\end{aligned}
$$

In the latter formula we made approximation

$$
\langle\widetilde{W} \widetilde{W} G\rangle \rightarrow\langle\widetilde{W} \widetilde{W}\rangle\langle G\rangle
$$

The value $\left\langle\widetilde{W}_{\mathbf{p}, \sigma}\right\rangle_{\text {conf }}=0$ because in initial Hamiltonian in the sum over $\mathbf{p}$ the term with $\mathbf{p}=\mathbf{0}$ is absent. The configurational averaged value is equal to

$$
\left\langle\widetilde{W}_{\mathbf{p}, \sigma} \widetilde{W}_{\mathbf{p}^{\prime}, \sigma}\right\rangle_{\mathrm{conf}}=\frac{1}{N} S_{2}(\mathbf{p}) \widetilde{w}_{\mathbf{p}, \sigma} \widetilde{w}_{\mathbf{p}^{\prime}, \sigma} \delta_{\mathbf{p},-\mathbf{p}^{\prime}}
$$

where $S_{2}(\mathbf{p})=\left\langle\varrho_{\mathbf{p}} \varrho_{-\mathbf{p}}\right\rangle$ is pair structural factor. From the equation for the averaged Green function in such an approach, the final expression is obtained

$$
\mathcal{G}(\mathbf{k}, \mathbf{q} \mid \omega) \equiv\left\langle G^{\sigma}(\mathbf{k}, \mathbf{q} \mid \omega)\right\rangle_{\mathrm{conf}}=\frac{\delta_{\mathbf{k}, \mathbf{q}}}{2 \pi} \frac{1}{\left(\hbar \omega-\mathcal{E}_{\mathbf{k}, \sigma}-\Sigma_{\sigma}(\mathbf{k} \mid \omega)\right)} .
$$

The self-energy part of the one-electron Green function in this approximation is

$$
\Sigma_{\sigma}(\mathbf{k} \mid \omega)=\frac{1}{N} \sum_{\mathbf{p} \in \Lambda} \frac{S_{2}(\mathbf{p})\left|\widetilde{w}_{\mathbf{p}, \sigma}\right|^{2}}{\hbar \omega-\mathcal{E}_{\mathbf{k}-\mathbf{p}, \sigma}}
$$

Equation for electron spectrum in Stonner subband with spin orientation $\sigma$ takes the form

$$
\hbar \omega=\mathcal{E}_{\mathbf{k}, \sigma}+\frac{1}{N} \sum_{\mathbf{p} \in \Lambda} \frac{S_{2}(\mathbf{p})\left|\widetilde{w}_{\mathbf{p}, \sigma}\right|^{2}}{\hbar \omega-\mathcal{E}_{\mathbf{k}-\mathbf{p}, \sigma}} .
$$

The electron energy in the long-wavelength region (small $\mathbf{k}$ )

$$
E(k)=\Delta(h)+\frac{\hbar^{2} k^{2}}{2 m^{*}}+\ldots,
$$

where $m^{*}$ is the effective electron mass in the external magnetic field

$$
m^{*}=\frac{m}{1+\frac{16 \Omega_{0} m^{2}}{3 \pi^{2} \hbar^{2}} \int_{0}^{\infty} \frac{\mathrm{d} p_{1}}{p_{1}^{2}} S_{2}\left(p_{1}\right)\left|\widetilde{w}_{p_{1}, \sigma}\right|^{2}}
$$

and

$$
\Delta(h)=\frac{2 \Omega_{0} m}{\pi^{2} \hbar^{2}} \int_{0}^{\infty} \mathrm{d} p_{1} S_{2}\left(p_{1}\right)\left|\widetilde{w}_{p_{1}, \sigma}\right|^{2}-\varkappa_{\sigma} \mu_{\mathrm{B}} h
$$


is Stonner gap in the electron spectrum.

For non-perturbation case when $\Sigma_{\sigma}(\mathbf{k} \mid \omega) \equiv 0$ the well known formula is obtained

$$
\varrho^{\sigma}(\omega)=\frac{m^{3 / 2}}{\sqrt{2} \pi^{2} \hbar^{3}} \sqrt{\hbar \omega+\varkappa_{\sigma} \mu_{\mathrm{B}} h}
$$

which describes the density of states of free electrons in the external magnetic field.

In the $\Sigma_{\sigma}(\mathbf{k} \mid \omega) \neq 0$ case the self-energy part contains the real and imaginary parts

$$
\Sigma_{\sigma}(\mathbf{k} \mid \omega)=\Sigma^{\prime}(\mathbf{k} \mid \omega)+\mathrm{i} \Sigma^{\prime \prime}(\mathbf{k} \mid \omega)
$$

where

$\Sigma^{\prime}(\mathbf{k} \mid \omega)=\frac{1}{N} \mathcal{P} \sum_{\mathbf{p} \in \Lambda} \frac{S_{2}(\mathbf{p})\left|\widetilde{w}_{\mathbf{p}, \sigma}\right|^{2}}{\hbar \omega-\mathcal{E}_{\mathbf{k}-\mathbf{p}, \sigma}}, \quad \Sigma^{\prime \prime}(\mathbf{k} \mid \omega)=-\frac{\pi}{N} \sum_{\mathbf{p} \in \Lambda} S_{2}(\mathbf{p})\left|\widetilde{w}_{\mathbf{p}, \sigma}\right|^{2} \delta\left(\hbar \omega-\mathcal{E}_{\mathbf{k}-\mathbf{p}, \sigma}\right)$.

Equation for density of states may be written in such a form

$$
\begin{aligned}
& \varrho^{\sigma}(E)=\left.\frac{m}{2 \pi^{2} \hbar} \sum_{i} \widetilde{p}_{i}\left|-1-\frac{\Omega_{0} m^{2}}{4 \pi^{2} \hbar^{4}} \frac{1}{\widetilde{p}_{i}^{3}} \int_{0}^{\infty} \mathrm{d} p p S_{2}(p)\right| \widetilde{w}_{p, \sigma}\right|^{2} \ln \left|\frac{E_{\sigma}-\frac{\hbar^{2}}{2 m}\left(\widetilde{p}_{i}-p\right)^{2}}{E_{\sigma}-\frac{\hbar^{2}}{2 m}\left(\widetilde{p}_{i}+p\right)^{2}}\right| \\
& -\left.\frac{\Omega_{0} m^{2}}{4 \pi^{2} \hbar^{4}} \frac{1}{\widetilde{p}_{i}} \int_{0}^{\infty} \mathrm{d} p p S_{2}(p)\left|\widetilde{w}_{p, \sigma}\right|^{2}\left(\frac{p / \widetilde{p}_{i}-1}{E_{\sigma}-\frac{\hbar^{2}}{2 m}\left(\widetilde{p}_{i}-p\right)^{2}}+\frac{p / \widetilde{p}_{i}+1}{E_{\sigma}-\frac{\hbar^{2}}{2 m}\left(\widetilde{p}_{i}+p\right)^{2}}\right)\right|^{-1} \cdot(30)
\end{aligned}
$$

In this equation $E_{\sigma}=E+\varkappa_{\sigma} \mu_{\mathrm{B}} h$, and $\widetilde{p}_{i}$ denotes simple roots of equation

$$
E_{\sigma}-\frac{\hbar^{2}}{2 m} \widetilde{p}^{2}-\frac{\Omega_{0} m}{4 \pi^{2} \hbar^{2}} \frac{1}{\widetilde{p}} \int_{0}^{\infty} \mathrm{d} p p S_{2}(p)\left|\widetilde{w}_{p, \sigma}\right|^{2} \ln \left|\frac{E_{\sigma}-\frac{\hbar^{2}}{2 m}(\widetilde{p}-p)^{2}}{E_{\sigma}-\frac{\hbar^{2}}{2 m}(\widetilde{p}+p)^{2}}\right|=0 .
$$

If we don't employ approach (23), and start with the iteration procedure, then the expression for the averaged function of configurations will be written in series form

$$
\begin{aligned}
& \left(\hbar \omega-\mathcal{E}_{\mathbf{k}, \sigma}\right) \mathcal{G}(\mathbf{k} \mid \omega)=\frac{1}{2 \pi}\left\{1+\frac{1}{N} \sum_{\mathbf{p}_{\mathbf{1}} \in \Lambda} \frac{\left|\widetilde{w}_{\mathbf{p}_{\mathbf{1}}, \sigma}\right|^{2} S_{2}\left(\mathbf{p}_{\mathbf{1}}\right)}{\left(\hbar \omega-\mathcal{E}_{\mathbf{k}-\mathbf{p}_{\mathbf{1}}, \sigma}\right)\left(\hbar \omega-\mathcal{E}_{\mathbf{k}, \sigma}\right)}\right. \\
& \quad+\frac{1}{N^{2}} \sum_{\mathbf{p}_{\mathbf{1}}, \mathbf{p}_{\mathbf{2}} \in \Lambda} \frac{\left|\widetilde{w}_{\mathbf{p}_{1}, \sigma}\right|^{2}\left|\widetilde{w}_{\mathbf{p}_{2}, \sigma}\right|^{2} S_{2}\left(\mathbf{p}_{\mathbf{1}}\right) S_{2}\left(\mathbf{p}_{\mathbf{2}}\right)}{\left(\hbar \omega-\mathcal{E}_{\mathbf{k}-\mathbf{p}_{\mathbf{1}}, \sigma}\right)\left(\hbar \omega-\mathcal{E}_{\mathbf{k}-\mathbf{p}_{\mathbf{1}}-\mathbf{p}_{\mathbf{2}}, \sigma}\right)\left(\hbar \omega-\mathcal{E}_{\mathbf{k}-\mathbf{p}_{\mathbf{2}}, \sigma}\right)\left(\hbar \omega-\mathcal{E}_{\mathbf{k}, \sigma}\right)} \\
& \quad+\frac{1}{N^{2}} \sum_{\mathbf{p}_{\mathbf{1}}, \mathbf{p}_{\mathbf{3}} \in \Lambda} \frac{\left|\widetilde{w}_{\mathbf{p}_{\mathbf{1}}, \sigma}\right|^{2}\left|\widetilde{w}_{\mathbf{p}_{\mathbf{3}}, \sigma}\right|^{2} S_{2}\left(\mathbf{p}_{\mathbf{1}}\right) S_{2}\left(\mathbf{p}_{\mathbf{3}}\right)}{\left(\hbar \omega-\mathcal{E}_{\mathbf{k}-\mathbf{p}_{\mathbf{1}}, \sigma}\right)\left(\hbar \omega-\mathcal{E}_{\mathbf{k}, \sigma}\right)\left(\hbar \omega-\mathcal{E}_{\mathbf{k}-\mathbf{p}_{\mathbf{3}}, \sigma}\right)\left(\hbar \omega-\mathcal{E}_{\mathbf{k}, \sigma}\right)} \\
& \left.\quad+\frac{1}{N^{2}} \sum_{\mathbf{p}_{\mathbf{1}}, \mathbf{p}_{\mathbf{2}} \in \Lambda} \frac{\left|\widetilde{w}_{\mathbf{p}_{\mathbf{1}}, \sigma}\right|^{2}\left|\widetilde{w}_{\mathbf{p}_{\mathbf{2}}, \sigma}\right|^{2} S_{2}\left(\mathbf{p}_{\mathbf{1}}\right) S_{2}\left(\mathbf{p}_{\mathbf{2}}\right)}{\left(\hbar \omega-\mathcal{E}_{\mathbf{k}-\mathbf{p}_{\mathbf{1}}, \sigma}\right)\left(\hbar \omega-\mathcal{E}_{\mathbf{k}-\mathbf{p}_{\mathbf{1}}-\mathbf{p}_{\mathbf{2}}, \sigma}\right)\left(\hbar \omega-\mathcal{E}_{\mathbf{k}-\mathbf{p}_{\mathbf{1}}, \sigma}\right)\left(\hbar \omega-\mathcal{E}_{\mathbf{k}, \sigma}\right)}+\ldots\right\}
\end{aligned}
$$


We can see, that Green function is represented in the form of infinite series, each following term of which contains an additional factor $|\widetilde{w}|^{2} S_{2} / N(\hbar \omega-\varepsilon)(\hbar \omega-\varepsilon)$ with suitable combination indexes. The issue of convergence of such series remains open, because in our problem small parameters are absent.

Such series in field theory are very well known. Especially it is frequently met in the problems concerning the interaction of electrons with bosonic field (electronphonon interaction). But in the field models, the $S_{2}(\mathbf{q} \mid \omega)$ and $\widetilde{w}_{\mathbf{I}, \sigma}$ values have got another sense. Therefore we can use the results known from the field theory for presenting the self-energy part of one-electron Green function

$$
\Sigma(\mathbf{k} \mid \omega)=\frac{1}{N} \sum_{\mathbf{p} \in \Lambda} \frac{S_{2}(\mathbf{p})\left|\widetilde{w}_{\mathbf{p}, \sigma}\right|^{2}}{\hbar \omega-\mathcal{E}_{\mathbf{k}-\mathbf{p}, \sigma}-\Sigma(\mathbf{k}-\mathbf{p} \mid \omega)} .
$$

The absence of a small parameter in our problem does not permit to estimate a correct approximation for self-energy part. The detailed numerical computations and studies are necessary.

For self-consistent computation of the dispersion law it is necessary to find equations for calculation magnetization $M$ of system localized spins and electron spin polarization $m$. Such equations were obtained in the approach of self-consistent field and their form is written in [6]

$$
\begin{aligned}
M & =B_{\mathrm{s}}\left(\frac{S}{T}\left[\mu h+c S M J_{0}+m \frac{V}{N} I_{0}\right]\right), \\
\frac{8}{3}\left(\frac{E_{\mathrm{F}}}{T}\right)^{3 / 2} m & =F_{1 / 2}\left(\frac{\mu+\xi}{T}\right)-F_{1 / 2}\left(\frac{\mu-\xi}{T}\right), \\
\frac{4}{3}\left(\frac{E_{\mathrm{F}}}{T}\right)^{3 / 2} & =F_{1 / 2}\left(\frac{\mu+\xi}{T}\right)+F_{1 / 2}\left(\frac{\mu-\xi}{T}\right) .
\end{aligned}
$$

Here $F_{1 / 2}(\alpha)$ is the Fermi-Dirak integral. Values $I_{0}=\lim _{\mathbf{k} \rightarrow \mathbf{0}} I_{\mathbf{k}}, \quad J_{0}=\lim _{\mathbf{k} \rightarrow \mathbf{0}} J_{\mathbf{k}}$ and $\mu$ is the chemical potential, and variable $\xi=\mu h+\frac{1}{2} c I_{0} S M$. In the first equation of the system we employed the notation $B_{\mathrm{s}}(x)$ for Brillouin function. A solution of the system (33) makes it possible to build the dependence $M$ and $m$ on temperature at different parameters values $J_{0}, I_{0}, n, S, E_{\mathrm{F}}$.

From equations (33) at $T=0$, the value of electron spin polarization $m$ is smaller than maximal possible value which is equal to 1 . It is valid for $S\left|I_{0}\right| / 2 E_{\mathrm{F}}$ parameter considerably smaller for unit. Condition $S\left|I_{0}\right| / 2 E_{\mathrm{F}} \ll 1$ is also valid for all ferromagnetic metals and semiconductors. It is so that $E_{\mathrm{F}}$ due to real electrons densities is a big value: $E_{\mathrm{F}} \sim 10^{4} \mathrm{~K}$. In this case, using the asymptotic for Fermi-Dirak function $F_{n}(\alpha)=\alpha^{n+1} /(n+1)$, the analytic expression for electron magnetization at temperature $T=0$ was obtained

$$
m=\frac{1}{2}\left[\left(1+\frac{c S I_{0} M}{2 E_{\mathrm{F}}}\right)^{3 / 2}-\left(1-\frac{c S I_{0} M}{2 E_{\mathrm{F}}}\right)^{3 / 2}\right] .
$$




\title{
4. Conclusions
}

In this paper firstly the equations for spectrum and electron states density of structurally disordered $s-d$ model are obtained. The dispersion law of both Stonner subbands is investigated. The electron effective mass in the first Born approximation is found. The integral equation for self-energy part is obtained for special structure type. It is valid in long-wavelength region.

The system of self-consistent equations for the model of magnet states calculation is obtained. If $I_{0}>0$ (ferromagnetic spin-electron interaction) then $m$ in (34) will be positive and if $I_{0}<0$ (non-ferromagnetic spin-electron interaction) then $m$ is negative. In the first case, spin and electron magnetization will be co-directed and in the second case controversially directed and partly compensate one another. Particularly, when parameter $S I_{0} / 2 E_{\mathrm{F}}$ is close to a unit, $m$ also approaches a unit. In case $S I_{0} / 2 E_{\mathrm{F}} \geqslant 1$ value $m$ is identically equal to unit. The last two cases may be realized in magnetic semiconductors with small density of conductivity electrons.

\section{References}

1. Nagajev E.L. Physics of magnetic semiconductors. - Moscow, Nauka, 1979.

2. Fisher K.H. Spin glasses (I). // Phys. Stat. Sol. (b), 1983, vol. 116, No. 2, p. 357-414.

3. Tselvick A.M., Wiegmann P.B. Exact results in the theory of magnetic alloys. // Advances in Phys., 1983, vol. 32, No. 4, p. 453-713.

4. Andrej N., Zuruja K., Löwenstein J.H. Solution of the Kondo problem. // Rew. Mod. Phys., 1983, vol. 32, No. 2, p. 331-402.

5. Yonezawa I.A., Morigaki K. Coherent potential approximation. // Prog. Theor. Phys. (Supplement), 1973, vol. 58, p. 1-76.

6. Rudavsky Yu.K., Ponedilok G.V., Dorosh L.A. Long-wave spin excitation in crystalline s-d models. // Cond. Matter Phys., 1998, vol. 1(13), p. 145-160.

\section{Спектр та густина електронних станів невпорядкованої $s$ - $d$ моделі}

\author{
Ю.Рудавський, Г.Понеділок, Л.Дорош
}

Національний університет "Львівська політехніка"

79013 Львів, вул. С.Бандери, 12

Отримано 23 серпня 2000 р., в остаточному вигляді 1 листопада 2000 р.

Досліджується структурно невпорядкована s-d модель магнетизму металів. Запропонована спрощена модель структури бінарної суміші, пораховані структурні кореляційні функції. Отримані рівняння для самоузгодженого розрахунку спектра, густини одноелектронних станів, намагніченості, електронної спінової поляризації і температури фазового переходу

Ключові слова: аморфні системи, намагніченість, поляризація

PACS: $75.30 . D s, 75.50 . K$ 